\title{
Thermodynamic Laws and Equipartition Theorem in Relativistic Brownian Motion
}

\author{
T. Koide and T. Kodama \\ Instituto de Física, Universidade Federal do Rio de Janeiro, \\ C.P. 68528, 21941-972, Rio de Janeiro, Brazil
}

\begin{abstract}
We extend the stochastic energetics to a relativistic system. The thermodynamic laws and equipartition theorem are discussed for a relativistic Brownian particle and the first and the second law of thermodynamics in this formalism are derived. The relation between the relativistic equipartition relation and the rate of heat transfer is discussed in the relativistic case together with the nature of the noise term.
\end{abstract}

\section{INTRODUCTION}

Physical interpretation and theoretical interpretation of Brownian motion, a well-known process named after the Scottish botanist Robert Brown in 1827, were first established by Einstein and Smoluchowski, then developed by Langevin and many others. From these studies, it was made clear that Brownian motion is nothing but the manifestation of the presence of invisible microscopic bodies such as atoms and molecules, and their thermal motion. Thus, Brownian motion has naturally been discussed in relation with kinetic theories and thermodynamics. Nowadays concepts and methods of stochastic processes introduced in the formulation of Brownian motion are widely applied to various fields of science such as biophysics and economy. Mathematical foundation of these methods has also been well studied.

After thermodynamics was established in the nineteenth century, many efforts have been made to understand the thermodynamic principles from the microscopic point of view. The achievements of these efforts are summarized today as statistical mechanics. There, the time average of microscopic behaviors is replaced by an average in terms of a suitably chosen statistical ensemble of microscopic events and the temporal behavior of each particle is not questioned. Therefore, the basic question to the foundation of statistical mechanics is how this substitution of averages is justified from the microscopic deterministic point of view. 
Of course such a question has been studied deeply in many ways since the time of Maxwell, Boltzmann and Gibbs such as $H$-theorem and ergodic theory. Mathematically rigorous or not, for physicists the overwhelming success of statistical mechanics itself to describe the nature for thermally equilibrated systems could be considered as the proof of validity of the hypothesis.

On the other hand, recently, it is more and more becoming important to understand the bulk properties of a dynamical system where the thermodynamic equilibrium is not necessarily attained. For example, in the field of relativistic heavy ion collisions (RIC), we would like to determine the thermodynamic properties of quark and gluon plasma (QGP) from the experimental data. RIC is somehow the unique way in laboratories to extract the thermodynamic properties of Quantum Chromodynamics (QCD) in the deconfined phase. However, of course, real systems in RIC situation are not in thermodynamic equilibrium, since the system size and the reaction time scale are finite. Although many successful models, such as relativistic hydrodynamics have been employed to describe several aspects of the observed data, we feel still an existence of "missing link" between the thermodynamic properties and microscopic dynamics. For example, several questions like, "How much can we be sure that the equation of state used to fit the experimental flow data with hydrodynamics should be the same as that of obtained from the lattice QCD calculations?", "Is the extended thermodynamics valid and unique?", or "How to deal with the relativistic covariance and thermodynamic limit?" are yet difficult to be answered. In this sense, it may still worthwhile to ask the repeated question for the basic foundation of statistical mechanics from the view point of these new physics necessities of nowadays.

The stochastic energetics may serve a useful tool to understand the questions above [1]. In this approach, the dynamics of each event of a Brownian motion is directly reflected in the change of thermodynamic quantities, such as heat, energy and work. That is, these thermodynamic quantities are also dealt as stochastic variables, reflecting in the name of this approach, stochastic energetics. Therefore, the application of this approach seems to be useful for RIC studies, where event by event fluctuations are considered to carry important information on QCD [2].

So far, the stochastic energetics has been applied to non-relativistic phenomena. However it seems very attractive to apply this approach to investigate the thermodynamic properties for relativistic systems. This is because it is possible to incorporate the effect of special 
relativity (such as the Lorentz transform) into the model in a straight-forward manner, permitting to discuss directly the relation between thermodynamics and the theory of relativity. Such an analysis is difficult in statistical physics where temporal averages are replaced by ensemble averages from the beginning. The purpose of this paper is to show that the stochastic energetics is still applicable to Brownian motion of a relativistic particle. It is important to notice that this is not a trivial problem, because equations of motion for stochastic variables involves a finite time interval so that the space and time entangles when the Lorentz transformation is involved. Therefore, we have to pay a special attention for the treatment of noise when we discuss a relativistic stochastic motion in a general reference frame.

The present paper is organized as follows: In Sec. 2, we introduce a model of relativistic Brownian motion. Relativistic generalization of Brownian motion has been discussed by many authors [3 15], but the definitive answer to this problem has not yet established, or simply does not exist in a unique manner. As mentioned above, this is because of the difficulty to introduce a manifestly Lorentz covariant noise in an unambiguous way, as well as its physical model. Here, instead of formulating a covariant equation of motion for stochastic variables in a general case, we discuss a less ambitious problem. We start from a Langevin equation for relativistic Brownian motion defined in the rest frame of a heat bath. Then we determine how the noise term should transform under an arbitrary Lorentz boost of the system in order to keep the internal consistency. We show that in such a situation, we can introduce a consistent noise term. In Sec. 3, we apply the idea of the stochastic energetics to our model of relativistic Brownian motion and derive the first and the second law of thermodynamics. We also discuss the relation between the rate of heat transfer and the relativistic equipartition relation. Summary and concluding remarks are given in Sec. 4 .

In this paper, we used the natural unit, $\hbar=c=k_{B}=1$.

\section{MODEL OF RELATIVISTIC BROWNIAN MOTION}

As mentioned in the introduction, the formulation of the relativistic Langevin equation contains still an open question due to the transformation property of the thermal noise. To avoid this question, we start with a well-defined model of relativistic Brownian motion in the rest frame of a heat bath. Then we study the transformation of the system under a Lorentz boost and check the consistency between our model and special relativity. 


\section{A. Relativistic Brownian motion in the rest frame of heat bath}

We consider the Brownian motion of a relativistic particle with mass $m$ in the $3+1$ dimension. In the rest frame of a heat bath, we consider the following Langevin equation,

$$
\begin{aligned}
\frac{d \mathbf{x}^{*}}{d t^{*}} & =\frac{\mathbf{p}^{*}}{p^{0 *}}, \\
\frac{d \mathbf{p}^{*}}{d t^{*}} & =-\nu\left(p^{0 *}\right) \mathbf{p}^{*}+\sqrt{2 D\left(p^{0 *}\right)} \mathbf{N}\left(t^{*}\right),
\end{aligned}
$$

where $p^{0 *}=\sqrt{\left(p^{*}\right)^{2}+m^{2}}$, that is, the particle always kept on the mass-shell. Throughout this paper, we use the symbol * to indicate the value of a variable defined in the rest frame of the heat bath. The parameters $\nu\left(p^{0 *}\right)$ and $D\left(p^{0 *}\right)$ characterize, respectively, the relaxation of the momentum and the strength of the noise. We assume that they are Lorentz scalar functions, depending only on the particle energy in this frame. $\mathbf{N}$ is a Gaussian white noise three-vector

$$
\mathbf{N}\left(t^{*}\right)=\left(\begin{array}{c}
N^{1} \\
N^{2} \\
N^{3}
\end{array}\right),
$$

and has the following correlation properties,

$$
\begin{aligned}
\left\langle\mathbf{N}\left(t^{*}\right)\right\rangle_{\mathrm{RF}} & =0 \\
\left\langle N^{i}\left(t^{*}\right) N^{j}\left(t^{*^{\prime}}\right)\right\rangle_{\mathrm{RF}} & =\delta_{i j} \delta\left(t^{*}-t^{*^{\prime}}\right) .
\end{aligned}
$$

The symbol $\langle X\rangle_{\mathrm{RF}}$ denotes the stochastic average of $X$ in the rest frame of the heat bath (we refer to as RF). The similar Langevin equations have already discussed in Refs. [10 15]. Note that the Langevin equation can be obtained even from a binary collision model [16].

Now we replace the Langevin equation with the following stochastic differential equation $(\mathrm{SDE})$,

$$
\begin{aligned}
& d \mathbf{x}^{*}=\frac{\mathbf{p}^{*}}{p^{0 *}} d t^{*}, \\
& d \mathbf{p}^{*}=-\nu\left(p^{0 *}\right) \mathbf{p}^{*} d t^{*}+\sqrt{2 D\left(p^{0 *}\right)} d \mathbf{w}_{t^{*}} .
\end{aligned}
$$

Here we have introduced the Wiener process $\mathbf{w}_{t^{*}}$ and its difference by

$$
d \mathbf{w}_{t^{*}} \equiv \mathbf{w}_{t^{*}+d t^{*}}-\mathbf{w}_{t^{*}}=\mathbf{N}\left(t^{*}\right) d t^{*}
$$


The correlations are given by

$$
\begin{aligned}
\left\langle d \mathbf{w}_{t_{i}^{*}}^{*}\right\rangle_{\mathrm{RF}} & =0, \\
\left\langle d \mathbf{w}_{t_{k}^{*}}^{i *} d \mathbf{w}_{t_{l}^{*}}^{j *}\right\rangle_{\mathrm{RF}} & =d t^{*} \delta_{i j} \delta_{k l} .
\end{aligned}
$$

The last term of Eq. (5b),

$$
\sqrt{2 D\left(p^{0 *}\right)} d \mathbf{w}_{t^{*}}^{*}
$$

is a kind of Stieltjes integral for the stochastic variable. The definition of Stieltjes integral in stochastic variables is known to be not unique. Here we consider the three typical cases. Note that this problem associated with the discretization scheme is discussed in Refs. [12, 13] in detail.

1. Ito interpretation [17]:

In this case, the term (9) is interpreted as

$$
\sqrt{2 D\left(p^{0 *}\right)} \circledast d \mathbf{w}_{t^{*}}^{*}=\sqrt{2 D\left(p^{0 *}\left(t^{*}\right)\right)}\left(\mathbf{w}_{t^{*}+d t^{*}}^{*}-\mathbf{w}_{t^{*}}^{*}\right) .
$$

Hereafter, we use the symbol $\circledast$ to indicate the Ito interpretation of the integral measure.

2. Stratonovich-Fisk interpretation [17]:

In this case (hereafter, we use the symbol o), the term (9) is interpreted as

$$
\begin{aligned}
\sqrt{2 D\left(p^{0 *}\right)} \circ d \mathbf{w}_{t^{*}}^{*} & =\frac{\left.\sqrt{2 D\left(p^{0 *}\left(t^{*}+d t^{*}\right)\right)}+\sqrt{2 D\left(p^{0 *}\left(t^{*}\right)\right.}\right)}{2}\left(\mathbf{w}_{t^{*}+d t^{*}}^{*}-\mathbf{w}_{t^{*}}^{*}\right) \\
& =\sqrt{D\left(p^{0 *}\right)} \partial_{\mathbf{p}^{*}} \sqrt{D\left(p^{0 *}\right)} d t^{*}+\sqrt{2 D\left(p^{0 *}\right)} \circledast d \mathbf{w}_{t^{*}}^{*} .
\end{aligned}
$$

where, in the second line, we used the Ito formula (See Appendix) to convert the Stratonovich-Fisk interpretation by the Ito scheme.

3. Hänggi-Klimontovich [16, 18, 20]:

In this case (hereafter, we use the symbol $\star$ ), the term (9) as

$$
\begin{aligned}
\sqrt{2 D\left(p^{0 *}\right)} \star d w_{t^{*}}^{*} & =\sqrt{2 D\left(p^{0 *}\left(t^{*}+d t^{*}\right)\right)}\left(\mathbf{w}_{t^{*}+d t^{*}}^{*}-\mathbf{w}_{t^{*}}^{*}\right) \\
& =2 \sqrt{D\left(p^{0 *}\right)}\left(\partial_{\mathbf{p}^{*}} \sqrt{D\left(p^{0 *}\right)}\right) d t^{*}+\sqrt{2 D\left(p^{0 *}\right)} \circledast d \mathbf{w}_{t^{*}}^{*} .
\end{aligned}
$$

Here, again, we used the Ito formula to express this interpretation in terms of Ito scheme. See Appendix. 
The equilibrium distribution function described by using these SDEs depends on the integral schemes defined above. To see this, we introduce the probability density in the phase space defined in the thermal bath rest frame,

$$
\rho\left(\mathbf{x}^{*}, \mathbf{p}^{*}, t^{*}\right)=\left\langle\delta^{(3)}\left(\mathbf{x}-\mathbf{x}^{*}\left(t^{*}\right)\right) \delta^{(3)}\left(\mathbf{p}-\mathbf{p}^{*}\left(t^{*}\right)\right)\right\rangle_{\mathrm{RF}} .
$$

The time evolution of $\rho\left(\mathbf{x}^{*}, \mathbf{p}^{*}, t^{*}\right)$ is given by the Fokker-Planck equation in a unified way for the different integral schemes as

$$
\begin{aligned}
\partial_{t^{*}} \rho\left(\mathbf{x}^{*}, \mathbf{p}^{*}, t^{*}\right) & =-\sum_{i} \partial_{\mathbf{x}^{*}}^{i}\left(\frac{p^{i *}}{p^{0 *}} \rho\left(\mathbf{x}^{*}, \mathbf{p}^{*}, t^{*}\right)\right)+\sum_{i} \partial_{\mathbf{p}^{*}}^{i}\left(\nu\left(\mathbf{p}^{0 *}\right) p^{i *} \rho\left(\mathbf{x}^{*}, \mathbf{p}^{*}, t^{*}\right)\right) \\
& +\sum_{i} \partial_{\mathbf{p}^{*}}^{i}\left(D^{1-\alpha}\left(\mathbf{p}^{0 *}\right) \partial_{\mathbf{p}^{*}}^{i} D^{\alpha}\left(\mathbf{p}^{0 *}\right) \rho\left(\mathbf{x}^{*}, \mathbf{p}^{*}, t^{*}\right)\right)
\end{aligned}
$$

The values of the parameter $\alpha$ correspond to the different discretization schemes: $\alpha=0$ for the Hänggi-Klimontovich scheme, $\alpha=1 / 2$ for the Stratonovich-Fisk scheme, and $\alpha=1$ for the Ito scheme.

From this equation, we find that the corresponding equilibrium distribution (spatially homogeneous and static) is given by

$$
\rho_{\mathrm{eq}}\left(\mathbf{x}^{*}, \mathbf{p}^{*}\right) \propto \exp \left(-\int^{p^{0}\left(\mathbf{p}^{*}\right)} s d s \frac{\nu(s)}{D(s)}-\alpha \ln D\left(p^{0}\left(\mathbf{p}^{*}\right)\right)\right) .
$$

Since the phase space volume element $d \Gamma=d^{3} \mathbf{x}^{*} d^{3} \mathbf{p}^{*}$ should be an invariant measure under the Lorentz transformation of the reference frame, we conclude that this equilibrium distribution (13) should be a Lorentz scalar function, although the proof is not trivial at all [21, 22]. As we will see later, we can define the transformation property of the noise by using this fact.

\section{B. Relativistic Brownian motion in a moving frame}

We consider the reference frame which is moving with a constant velocity $\mathbf{V}$ with respect to the rest frame of the heat bath. We refer to as MF-moving frame. The four-momentum $d p^{\mu}$ in this frame is then given by the Lorentz transform of $d p^{* \mu}$ as

$$
\begin{aligned}
d p^{\mu} & =\Lambda(\mathbf{V}) d p^{* \mu} \\
& =\left(\begin{array}{cc}
\gamma(\mathbf{V}) & \beta(V) \mathbf{n}^{T} \gamma(\mathbf{V}) \\
\beta(V) \mathbf{n} \gamma(\mathbf{V}) & \gamma(\mathbf{V}) P_{\|}+Q_{\perp}
\end{array}\right)\left(\begin{array}{c}
d p^{0 *} \\
d \mathbf{p}^{*}
\end{array}\right),
\end{aligned}
$$


where $\gamma(\mathbf{V})=1 / \sqrt{1-\mathbf{V}^{2}}$ and $\beta(V)=|\mathbf{V}|$. The projection operators are defined by $P_{\|}=$ $\mathbf{n n}^{T}$ and $Q_{\perp}=1-P_{\|}$with $\mathbf{n}=\mathbf{V} /|\mathbf{V}|$. In the proceeding calculations, we assume that the particle is always on mass-shell $p^{0}=\sqrt{\mathbf{p}^{2}+m^{2}}$ during the whole stochastic process so that the stochastic variables, $d p^{0 *}$ and $d \mathbf{p}^{*}$ are not independent. We then have,

$$
\begin{aligned}
d p^{0 *}=d \sqrt{\mathbf{p}^{* 2}+m^{2}} & =\left\{\left(-\nu\left(p^{0 *}\right)+(1-\alpha) \frac{D^{\prime}\left(p^{0 *}\right)}{p^{0 *}}\right) \frac{\left(\mathbf{p}^{*}\right)^{2}}{p^{0 *}}\right. \\
& \left.+D\left(p^{0 *}\right)\left(\frac{3}{p^{0 *}}-\frac{\left(\mathbf{p}^{*}\right)^{2}}{\left(p^{0 *}\right)^{3}}\right)\right\} d t^{*}+\sqrt{2 D\left(p^{0 *}\right)} \frac{\mathbf{p}^{*}}{p^{0 *}} \circledast d \mathbf{w}_{t^{*}}^{*} .
\end{aligned}
$$

where $D^{\prime}(x)=d D(x) / d x$. Substituting this expression into Eq. (16), we obtain the SDE in the MF as

$$
\begin{aligned}
d \mathbf{p} & =\left(-\frac{\nu\left(u^{\mu} p_{\mu}\right) \gamma(V)\left(p^{0}-\beta(V) p_{\mathrm{V}}\right)}{p^{0}}+(1-\alpha) \frac{D^{\prime}\left(u^{\mu} p_{\mu}\right)}{p^{0}}\right)\left\{\mathbf{p}-\frac{\beta(V) m^{2} \mathbf{n}}{p^{0}-\beta(V) p_{\mathrm{V}}}\right\} d t \\
& +\beta \gamma(V) \frac{D\left(u^{\mu} p_{\mu}\right)}{p^{0}}\left(2+\frac{m^{2}}{\gamma^{2}(V)\left(p^{0}-\beta(V) p_{\mathrm{V}}\right)^{2}}\right) \mathbf{n} d t+\widehat{\mathbf{B}} \circledast d \mathbf{w}_{t^{*}}^{*},
\end{aligned}
$$

where $p_{\mathrm{V}}=\mathbf{n}^{T} \mathbf{p}$ and

$$
\widehat{\mathbf{B}}=\sqrt{2 D\left(u^{\mu} p_{\mu}\right)} \frac{\gamma^{-1}(V)}{p^{0}-\beta(V) p_{\mathrm{V}}}\left\{p^{0} P_{\|}+\gamma(V)\left(p^{0}-\beta(V) p_{\mathrm{V}}+\beta(V)\left(\mathbf{n} \mathbf{p}^{T}\right)\right) Q_{\perp}\right\} .
$$

Here $u^{\mu}$ is the four velocity $(\gamma, \gamma \mathbf{V})$, normalized as $u^{\mu} u_{\mu}=1$. The presence of a second rank tensor $\widehat{\mathbf{B}}$ indicates that the noise is no longer isotropic in the moving frame [23].

The last term is not yet transformed because it contains the noise $d \mathbf{w}_{t^{*}}^{*}$ which is defined only in the RF. We first introduce the stochastic noise which shows the property of the Gaussian white noise in the MF as

$$
\begin{aligned}
\left\langle d \mathbf{w}_{t^{*}}\right\rangle_{\mathrm{MF}} & =0, \\
\left\langle d \mathbf{w}_{t_{l}^{*}}^{i} d \mathbf{w}_{t_{m}^{*}}^{j}\right\rangle_{\mathrm{MF}} & =d t^{*} \delta_{i j} \delta_{l m},
\end{aligned}
$$

Here the symbol $\langle X\rangle_{M F}$ denotes the stochastic average of $X$ in the MF. As was assumed in previous works [3 13], we may consider that the correlations of the noise term are Lorentz invariant in the rest frame of the particle, and we could write

$$
\begin{aligned}
\left\langle d \mathbf{w}_{t}\right\rangle_{\mathrm{MF}} & =\left\langle d \mathbf{w}_{t^{*}}^{*}\right\rangle_{R F}=0, \\
\tilde{\gamma}(\mathbf{p})\left\langle d \mathbf{w}_{t_{k}}^{i} d \mathbf{w}_{t_{l}}^{j}\right\rangle_{\mathrm{MF}} & =\gamma\left(\mathbf{p}^{*}\right)\left\langle d \mathbf{w}_{t_{k}^{*}}^{i *} d \mathbf{w}_{t_{l}^{*}}^{j *}\right\rangle_{\mathrm{RF}}=d \tau \delta_{i j} \delta_{k l},
\end{aligned}
$$

where $d \tau$ is the proper time of the particle, $\gamma\left(\mathbf{p}^{*}\right)$ and $\tilde{\gamma}(\mathbf{p})$ are the Lorentz factors of the particle in the RF and in the MF, respectively. They are related through

$$
\tilde{\gamma}(\mathbf{p})=\left(\Lambda(\mathbf{V}) \Lambda\left(-\mathbf{p}^{*}\right)\right)^{00}=\gamma(\mathbf{V}) \gamma\left(\mathbf{p}^{*}\right)\left(1-\beta(V) \frac{p_{\mathrm{V}}^{*}}{p^{0 *}}\right),
$$


where, now $\gamma(\mathbf{V})$ is the Lorentz factor associated to the Lorentz transformation from the $\mathrm{RF}$ to the MF. In this case, we conclude that the transformation property of the noise is given by

$$
d \mathbf{w}_{t^{*}}^{*}=\sqrt{\frac{d t^{*}}{d t}} d \mathbf{w}_{t}=\sqrt{\gamma(\mathbf{p}) \tilde{\gamma}^{-1}\left(\mathbf{p}^{*}\right)} d \mathbf{w}_{t}=\gamma^{1 / 2}(V) \sqrt{\frac{p^{0}-\beta(V) p_{\mathrm{V}}}{p^{0}}} d \mathbf{w}_{t} .
$$

Although very reasonable, a proof of the above argument is not obvious. The reason is that the Stieltjes integral associated with the noise term is defined on the time span $d t$, so that $d \mathbf{w}_{t^{*}}^{*}$ is non-local in the time $t$. Thus the Lorentz transformation entangles with the integration scheme in the order of $d t$. Then the noise term itself is not necessarily covariant but can constitute a Lorentz vector only together with the force term. That is, the force part and the stochastic part could be mixed. This might be understood well from the argument of the kinetic derivation of hydrodynamics. In the rest frame of fluids, the velocities of molecules are completely random and all the kinetic energy of molecules are replaced by internal thermodynamic quantities. On the other hand, when fluids move, a part of velocities of molecules contributes to the collective flow of fluids. In this case, the first order correlation calculated in the MF of the noise term in the rest frame of the heat bath would not necessarily vanish,

$$
\left\langle d \mathbf{w}_{t^{*}}^{*}\right\rangle_{\mathrm{MF}} \neq 0
$$

even if $\left\langle d \mathbf{w}_{t^{*}}^{*}\right\rangle_{\mathrm{RF}}=0$.

Considering the finiteness of $d t$, let us write, instead of Eq. (25), the following more general transformation property of the noise,

$$
\begin{aligned}
d \mathbf{w}_{t^{*}}^{*} & =\sqrt{\frac{d t^{*}}{d t}} d \mathbf{w}_{t}+\mathbf{C}_{\mathbf{p}} d t \\
& =\gamma^{1 / 2}(V) \sqrt{\frac{p^{0}-\beta(V) p_{\mathrm{V}}}{p^{0}}} d \mathbf{w}_{t}+\mathbf{C}_{\mathbf{p}} d t .
\end{aligned}
$$

Here $\mathbf{C}_{\mathbf{p}} d t$ term entangles with the force term in the MF, which should be separated from the pure stochastic part $d \mathbf{w}_{t}$, satisfying Eqs. (20) and (21). With this definition and using the Ito formula, the Langevin equation is given by

$$
\begin{aligned}
d \mathbf{p} & =\left(-\frac{\nu\left(u^{\mu} p_{\mu}\right) \gamma(V)\left(p^{0}-\beta(V) p_{\mathrm{V}}\right)}{p^{0}}+(1-\alpha) \frac{D^{\prime}\left(u^{\mu} p_{\mu}\right)}{p^{0}}\right)\left\{\mathbf{p}-\frac{\beta(V) m^{2} \mathbf{n}}{p^{0}-\beta(V) p_{\mathrm{V}}}\right\} d t \\
+ & \beta(V) \gamma(V) \frac{D\left(u^{\mu} p_{\mu}\right)}{p^{0}}\left(2+\frac{m^{2}}{\gamma^{2}(V)\left(p^{0}-\beta(V) p_{\mathrm{V}}\right)^{2}}\right) \mathbf{n} d t+\widehat{\mathbf{B}} \mathbf{C}_{p} d t+\tilde{\mathbf{B}} \circledast d \mathbf{w}_{t},
\end{aligned}
$$


where

$$
\tilde{\mathbf{B}}=\sqrt{\frac{\gamma(V)\left(p^{0}-\beta(V) p_{\mathrm{V}}\right)}{p^{0}}} \widehat{\mathbf{B}} .
$$

The last term in Eq. (28) should be calculated according to the Ito scheme. Note that the Ito formula to convert the SDE from one scheme to the other must be used only after the transformation of the noise, Eq. (27).

The corresponding Fokker-Plank equation for the SDE (28) is

$$
\partial_{t} \rho=-\sum_{i} \partial_{x}^{i} \frac{p^{i}}{p^{0}} \rho+\sum_{i} \partial_{p}^{i}\left[-\mathbf{A}^{i}+\frac{1}{2} \partial_{\mathbf{p}}^{j}\left(\tilde{\mathbf{B}} \tilde{\mathbf{B}}^{T}\right)^{i j}\right] \rho,
$$

where

$$
\begin{aligned}
\mathbf{A} & =\left(-\frac{\nu\left(u^{\mu} p_{\mu}\right) \gamma(V)\left(p^{0}-\beta(V) p_{\mathrm{V}}\right)}{p^{0}}+(1-\alpha) \frac{D^{\prime}\left(u^{\mu} p_{\mu}\right)}{p^{0}}\right)\left\{\mathbf{p}-\frac{\beta(V) m^{2} \mathbf{n}}{p^{0}-\beta(V) p_{\mathrm{V}}}\right\} \\
& +\beta \gamma(V) \frac{D\left(u^{\mu} p_{\mu}\right)}{p^{0}}\left(2+\frac{m^{2}}{\gamma^{2}(V)\left(p^{0}-\beta(V) p_{\mathrm{V}}\right)^{2}}\right) \mathbf{n}+\widehat{\mathbf{B}} \mathbf{C}_{\mathbf{p}} .
\end{aligned}
$$

Consequently, the equilibrium distribution function is given by the solution of the following equation,

$$
\left[-\mathbf{A}^{i}+\frac{1}{2} \partial_{\mathbf{p}}^{j}\left(\tilde{\mathbf{B}} \tilde{\mathbf{B}}^{T}\right)^{i j}\right] \rho_{\mathrm{eq}}(\mathbf{x}, \mathbf{p})=0,
$$

leading to the equilibrium distribution $\rho_{\mathrm{eq}}$ satisfying

$$
\left(\partial_{\mathbf{p}}^{i} \rho_{\mathrm{eq}}\right) / \rho_{\mathrm{eq}}=2 \sum_{j k l}\left(\tilde{\mathbf{B}} \tilde{\mathbf{B}}^{T}\right)_{i j}^{-1}\left(\mathbf{A}^{j}-\frac{1}{2} \partial_{\mathbf{p}}^{k}\left(\tilde{\mathbf{B}} \tilde{\mathbf{B}}^{T}\right)^{j k}\right) .
$$

Since $\rho_{e q}$ should be a scalar function, the above expression must coincide with the Lorentz transform of the logarithmic derivative of the equilibrium distribution function obtained in the RF. A lengthy but straightforward calculation shows that $\rho_{e q}$ can be the Lorentz scalar only when

$$
\mathbf{C}_{\mathbf{p}} \equiv 0 \text {. }
$$

Thus, for the present model of a Brownian motion with the noise from a given heat bath, we conclude that the noise term transforms separately from the force term when we go to one reference frame to the other. That is, the transformation of the noise (25) is consistent with the special relativity, and the corresponding SDE in a MF should be given by Eq. (18). When the background does not satisfy this condition, it is not obvious if we can always assume $\mathbf{C}_{\mathbf{p}} \equiv 0$ from the beginning. Our discussion here is only for the thermal background is homogeneous and static. 


\section{Generalized fluctuation-dissipation relation}

So far, we have not specified the parameter of the SDE, but when we demand that the equilibrium distribution function should be given by the Jüttner distribution in a MF,

$$
\rho_{\text {eq }}=\text { Const. } \times e^{-u^{\mu} p_{\mu} / T},
$$

where $T$ denotes temperature. From this condition, the parameters of the SDE satisfy the following relation,

$$
\nu\left(u^{\mu} p_{\mu}\right)=\frac{1}{u^{\mu} p_{\mu}}\left(\frac{D\left(u^{\mu} p_{\mu}\right)}{T}-\alpha D^{\prime}\left(u^{\mu} p_{\mu}\right)\right),
$$

which is the generalized Einstein's fluctuation-dissipation relation of relativistic Brownian motion. Note that this relation depends on $\alpha$, showing the discretization scheme dependence when $D$ is not a constant.

\section{RELATIVISTIC STOCHASTIC ENERGETICS}

Once we have established the SDE for a relativistic particle embedded in a heat bath, we can discuss the thermodynamic property of the system defined as the ensemble of these relativistic Brownian particles. For this purpose, from now on, we discuss only in the rest frame of the heat bath,

$$
\begin{aligned}
& d \mathbf{x}^{*}=\frac{\mathbf{p}^{*}}{p^{0 *}} d t^{*}, \\
& d \mathbf{p}^{*}=-\nabla \phi d t^{*}-\nu\left(p^{0 *}\right) \mathbf{p}^{*} d t^{*}+\sqrt{2 D\left(p^{0 *}\right)} \circledast d \mathbf{w}_{t^{*}},
\end{aligned}
$$

where we have not assumed the generalized fluctuation-dissipation relation. For the sake of the discussion of the first law of thermodynamics, we introduced a scalar potential $\phi\left(\mathbf{x}^{*}, t^{*}\right)$ where the explicit time dependence of $\phi$ represents the effect of some external forces.

\section{A. The First Law of Thermodynamics}

Following Ref. [1], let us introduce heat as a stochastic variable associated with Brownian motion. Among the three contributions of the forces in Eq.(37b), the last two terms represent the interactions between a system and the heat bath. The first term represents 
the mechanical force through the scalar potential $\phi$. Thus the work done by the heat bath, that is, the heat transfer, $d^{\prime} Q$ to a Brownian particle is defined as[30]

$$
d^{\prime} Q=\left(-\nu\left(p^{0 *}\right) \mathbf{p}^{*}+\sqrt{2 D\left(p^{0 *}\right)} \cdot \frac{d \mathbf{w}_{t^{*}}}{d t^{*}}\right) \circ d \mathbf{x}^{*} .
$$

Using Eq. (37b) we can rewrite Eq.(38) as

$$
\begin{aligned}
d^{\prime} Q & =\left(\frac{d \mathbf{p}^{*}}{d t^{*}}+\nabla \phi\right) \circ d \mathbf{x}^{*} \\
& =d\left(p^{0 *}+\phi\right)-\frac{\partial \phi}{\partial t^{*}} d t^{*}
\end{aligned}
$$

Here, we identify the first term represents the change of the total energy of a Brownian particle $d E$ including its potential energy. The quantity $\left(\partial \phi / \partial t^{*}\right) d t^{*}$ in the last term is the change of the energy contained in the scalar potential $\phi$ due to the change in some external parameters contained in $\phi$. That is, it can be interpreted as the mechanical work $d^{\prime} W$ done by the external forces to the system. Thus, rewriting Eq.(39) as

$$
d^{\prime} Q=d E-d^{\prime} W
$$

we can view this as the first law of thermodynamics in the framework of the stochastic energetics, the generalization of Ref. [1] for relativistic Brownian motion. Note that this relation is satisfied for each event of Brownian motion [31].

\section{B. The Second Law}

In the above, we introduced the potential $\phi$ to identify clearly the role of external work in the first law of thermodynamics, and, there, the choice of the parameters of the SDE was arbitrary. In the following discussion of other thermodynamic relations, however, we will consider only the case where $\phi=0$ and impose the generalized fluctuation-dissipation theorem to the SDE.

For deriving the second law of thermodynamics in the stochastic energetics, we introduce Shannon's information entropy,

$$
S=-\int d \Gamma \rho\left(\mathbf{x}^{*}, \mathbf{p}^{*}, t^{*}\right) \ln \rho\left(\mathbf{x}^{*}, \mathbf{p}^{*}, t^{*}\right),
$$

where $\rho\left(\mathbf{x}^{*}, \mathbf{p}^{*}, t^{*}\right)$ is defined in Eq.(13) and satisfies the Fokker-Plank equation (14). The integration in Eq.(41) is taken over all the phase space volume. From these definitions for 
heat (38) and entropy (41), it is easy to show that the time derivatives of heat and entropy are related as

$$
T \frac{d S}{d t^{*}}-\frac{1}{T}\left\langle\frac{d^{\prime} Q}{d t^{*}}\right\rangle_{\mathrm{RF}}=\int d \Gamma \frac{T D\left(p^{0 *}\right)}{\rho}\left(\nabla_{p} \rho+\frac{1}{T} \frac{\mathbf{p}^{*}}{p^{0 *}} \rho\right)^{2},
$$

Since the right-hand side of the above equation is non-negative, we obtain the following inequality,

$$
\frac{d S}{d t^{*}}-\frac{1}{T}\left\langle\frac{d^{\prime} Q}{d t^{*}}\right\rangle_{\mathrm{RF}} \geq 0
$$

which is nothing but the second law of thermodynamics. For the non-relativistic case, see Ref. [1, 24]. An important fact is that, differently from the first law, the second law is satisfied only for the average heat, and can be violated for each event, as is the case of the fluctuation theorem.

\section{Relativistic Equipartition Theorem}

In the preceding section, we have shown that the first and the second laws of thermodynamics can be consistently derived by generalizing the stochastic energetics to relativistic Brownian motion. Let us apply this approach to discuss another example, the equipartion theorem.

In non-relativistic systems, the equipartion theorem tells us that the average kinetic energy of a particle of an ideal gas in equilibrium is equally distributed by $T / 2$ for each degree of freedom. For 3-dimensional mono-atomic gas, we have

$$
\left\langle\frac{\mathbf{p}^{2}}{2 m}\right\rangle_{t \rightarrow \infty}=\frac{3}{2} T
$$

where $\langle O\rangle_{t}$ denotes the expectation value of $O$ at the instant $t$, which can be expressed as

$$
\langle O\rangle_{t}=\int d \Gamma \rho(\mathbf{x}, \mathbf{p}, t) O(\mathbf{x}, \mathbf{p})
$$

with $\rho(\mathbf{x}, \mathbf{p}, t)$ is the solution of the Fokker-Plank equation corresponding to the following non-relativistic SDE,

$$
\begin{aligned}
& d \mathbf{x}=\frac{\mathbf{p}}{m} d t \\
& d \mathbf{p}=-\nu \frac{\mathbf{p}}{m} d t-\nabla_{x} U(\mathbf{x}) d t+\sqrt{2 D} \circledast d \mathbf{w}_{t},
\end{aligned}
$$


where $U(\mathbf{x})$ is a potential and $\nu=D / T$. Note that, the above average (45) using the solution of the Fokker-Planck equation is equivalent to the event average for the corresponding noise.

In the stochastic energetics, this relation for equipartition of energy is seen to be valid in more general situation if the average heat transfer vanishes even if the system is not in equilibrium [1]. To see this, we consider the case where $D$ is a constant so that the noise is additive. Then, the time derivative of the heat described by this SDE is given by

$$
\begin{aligned}
\left\langle\frac{d^{\prime} Q}{d t}\right\rangle & =-\frac{2 D}{m T} \int d \Gamma\left(\frac{\mathbf{p}^{2}}{2 m}-\frac{3}{2} T\right) \rho(\mathbf{x}, \mathbf{p}, t) \\
& =-\frac{2 D}{m T}\left(\left\langle\frac{\mathbf{p}^{2}}{2 m}\right\rangle_{t}-\frac{3}{2} T\right),
\end{aligned}
$$

where the left hand side average is the event average. One can see that the equipartition relation is satisfied, when the heat transfer disappears even $\rho$ is not the equilibrium distribution. Thus we may consider the condition of the null heat transfer leads to the equipartition relation in more generalized situation. We will apply this idea to relativistic Brownian motion.

The relativistic analogue is not trivial in the sense that there is no simple interpretation of the quantity involved [25]. The expectation value of relativistic kinetic energy, $K=$ $\sqrt{p^{2}+m^{2}}-m$ does not satisfy a simple relation such as Eq. (44). Tolman proposed to read Eq. (44) as [26, 27]

$$
\frac{1}{2}\left\langle\mathbf{p} \frac{\partial E}{\partial \mathbf{p}}\right\rangle_{\mathrm{eq}}=\frac{3}{2} T
$$

or equivalently,

$$
\left\langle\frac{\mathbf{p}^{2}}{2 p^{0}}\right\rangle_{\mathrm{eq}}=\frac{3}{2} T
$$

where the subscript $e q$ denotes the expectation value at equilibrium, that is, $t \rightarrow \infty$. The left-hand side of Eq. (48) is proportional to the average of $\mathbf{p} \cdot \mathbf{v}$, which is interpreted to be the momentum transfer rate than the kinetic energy itself.

As an application of the stochastic energetics for relativistic Brownian motion, we can derive the relativistic analogue of Eq. (47). Taking the noise average of Eq. (38), we obtain

$$
\begin{aligned}
\left\langle\frac{d^{\prime} Q}{d t^{*}}\right\rangle_{\mathrm{RF}} & =\left\langle\frac{d \mathbf{p}^{*}}{d t^{*}} \circ d \mathbf{x}^{*}\right\rangle_{\mathrm{RF}}=\left\langle d \mathbf{p}^{*} \circ \frac{d \mathbf{x}^{*}}{d t^{*}}\right\rangle_{\mathrm{RF}} \\
& =-\int d \Gamma\left\{\frac{D\left(p^{0 *}\right)}{T} \frac{\left(\mathbf{p}^{*}\right)^{2}}{\left(p^{0 *}\right)^{2}}-\left(\nabla_{p} \cdot \frac{D\left(p^{0 *}\right) \mathbf{p}^{*}}{p^{0 *}}\right)\right\} \rho\left(\mathbf{x}^{*}, \mathbf{p}^{*}, t^{*}\right) \\
& =-\frac{1}{T}\left\langle D\left(p^{0 *}\right) \frac{\left(\mathbf{p}^{*}\right)^{2}}{\left(p^{0 *}\right)^{2}}\right\rangle_{t^{*}}+\left\langle\nabla_{p} \cdot \frac{D\left(p^{0 *}\right) \mathbf{p}^{*}}{p^{0 *}}\right\rangle_{t^{*}}
\end{aligned}
$$


To derive this expression, we have used the relation,

$$
\frac{\mathbf{p}^{*}}{p^{0 *}} \circ d \mathbf{p}^{*}=d \mathbf{p}^{*} \circledast\left(\frac{\mathbf{p}^{*}}{p^{0 *}}+\frac{1}{2} d \frac{\mathbf{p}^{*}}{p^{0 *}}\right) .
$$

From Eq. (50) the null heat transfer rate leads to a relation,

$$
\frac{1}{T}\left\langle D\left(p^{0 *}\right) \frac{\left(\mathbf{p}^{*}\right)^{2}}{\left(p^{0 *}\right)^{2}}\right\rangle_{t^{*}}=\left\langle\nabla_{p} \cdot \frac{D\left(p^{0 *}\right) \mathbf{p}^{*}}{p^{0 *}}\right\rangle_{t^{*}},
$$

independent of $\rho$. We call the above equation as the generalized equipartition theorem for the relativistic Brownian motion. Note that this relation does not have the same form of the Tolman relation (49). Only when the multiplicative noise is chosen as $D \propto p^{0 *}$, Eq. (52) coincides with

$$
\left\langle\frac{\mathbf{p}^{2}}{2 p^{0}}\right\rangle_{t^{*}}=\frac{3}{2} T
$$

as in the non-relativistic case of Eq. (47) which is valid even in out of equilibrium. In the ultra-relativistic limit, this relation is reduced to

$$
\langle E\rangle_{t^{*}}=3 T
$$

where $E=p^{0 *}$ so that it is related to the specific heat of the system.

The meaning of the equipartition theorem is modified for more general cases. For example, suppose the noise has the energy dependence as $D \propto\left(p^{0 *}\right)^{q}$ with $q=$ const, then Eq.(52) becomes

$$
\left\langle E^{q}\right\rangle_{t^{*}}=T \times(q+2)\left\langle E^{q-1}\right\rangle_{t^{*}}
$$

in the ultra-relativistic limit, and

$$
\left\langle|\mathbf{p}|^{q+2}\right\rangle_{t^{*}}=\operatorname{Tm} \times(q+3)\left\langle|\mathbf{p}|^{q}\right\rangle_{t^{*}}
$$

in the non-relativistic Brownian motion (for non-relativistic case, Eq. (50) is still the general form of Eq. (47) when the noise is multiplicative, provided that $p^{0 *}$ and $D\left(p^{0 *}\right)$ are $m$ and $D\left(\mathbf{p}^{2}\right)$, respectively). These expressions show that the ratio of two moments of kinetic energy should be kept constant proportional to the temperature. In this sense we call Eq. (52) as the generalized equipartition theorem.

For $q=-2$ in Eq. (55) or $q=-3$ in Eq. (56), the left hand side is finte, but the pre-factor in the right hand side vanishes. This means that the average value in the right 
hand side in each equation diverges but the products

$$
\begin{gathered}
\lim _{q \rightarrow-2}(q+2)\left\langle E^{q-1}\right\rangle_{t^{*}}, \\
\lim _{q \rightarrow-3}(q+3)\left\langle|\mathbf{p}|^{q}\right\rangle_{t^{*}},
\end{gathered}
$$

have finite positive values and the equalities are still valid. This is easily checked for equilibrium cases, since we can calculate explicitly

$$
\begin{aligned}
\left\langle E^{q}\right\rangle_{\mathrm{eq}} & =\frac{4 \pi}{Z} T^{q+3} \Gamma(q+3), \\
\left\langle|\mathbf{p}|^{q+2}\right\rangle_{\mathrm{eq}} & =\frac{2 \pi}{Z}(2 m T)^{(q+5) / 2} \Gamma((q+5) / 2),
\end{aligned}
$$

where $Z$ is the normalization factor of the equilibrium distribution function and $\Gamma(x)$ is the Gamma function. From the recursion relation of the Gamma function, we obtain Eqs. (55) and (56). Of course these relations do not make sense for $q<-2$ in Eq.(55) or for $q<-3$ in Eq.(56), since the left had side (so the right hand side, too) diverges.

Note that the condition, Eq.(55) or Eq.(56), itself does not determine the distribution function. Of course, if we assume that there exists a distribution function, $f\left(E ; t^{*}\right)$, independent of $q$, satisfying Eq.(55) for any $q>2$, we can demonstrate that such a distribution function should be proportional to $\exp (-E / T)$, and similary for non-relativistic case. However, for a finite $t^{*}$, the distribution function still reflects the initial condition and depends on $q$.

Thus, as stated before, the null heat transfer condition does not mean that the system is in equilibrium, but inversely, the null heat transfer condition is naturally satisfied in thermal equilibrium for any choice of $D\left(p^{0 *}\right)$. For more general noise coefficient, this can be seen directly from Eq. (50) by re-expressing it as

$$
\left\langle\frac{d^{\prime} Q}{d t^{*}}\right\rangle_{\mathrm{RF}}=-\int d \Gamma\left\{\frac{D\left(p^{0 *}\right)}{T} \frac{\left(\mathbf{p}^{*}\right)^{2}}{\left(p^{0 *}\right)^{2}}+\left(\frac{D\left(p^{0 *}\right) \mathbf{p}^{*}}{p^{0 *}}\right) \cdot\left(\nabla_{p} \ln \rho\left(\mathbf{x}^{*}, \mathbf{p}^{*}, t^{*}\right)\right)\right\} \rho\left(\mathbf{x}^{*}, \mathbf{p}^{*}, t^{*}\right) .
$$

The integrand vanishes if $\rho$ is given by the Jüttner distribution function. Therefore, in thermal equilibrium, both of the generalized equipartition theorem (52) and the Tolman relation (49) are satisfied at the same time.

To see how the null heat transfer condition is attained, we consider the case of a pion $m=139 \mathrm{MeV}$ in a heat bath of $T=150 \mathrm{MeV}$ as an example for a possible application to 


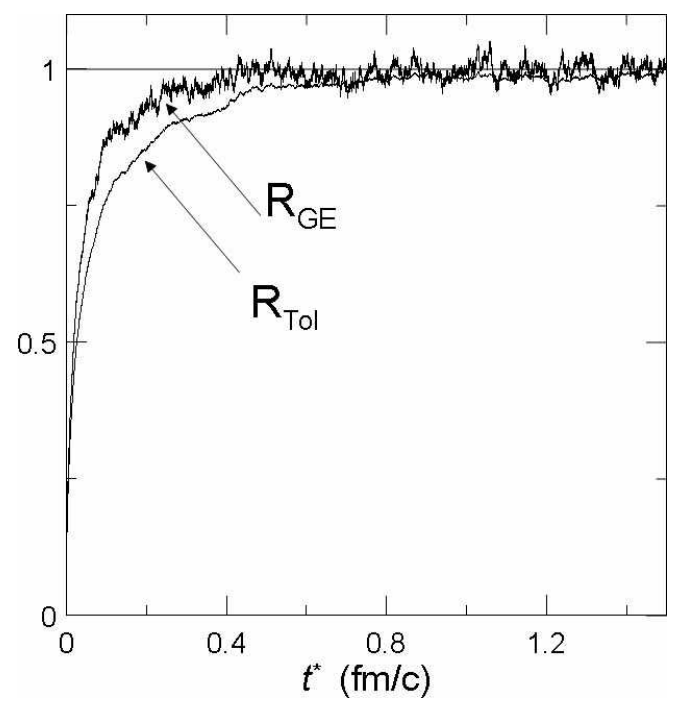

FIG. 1: The two ratios as a function of time $t^{*}$ with the initial condition of $50 \mathrm{MeV}$.

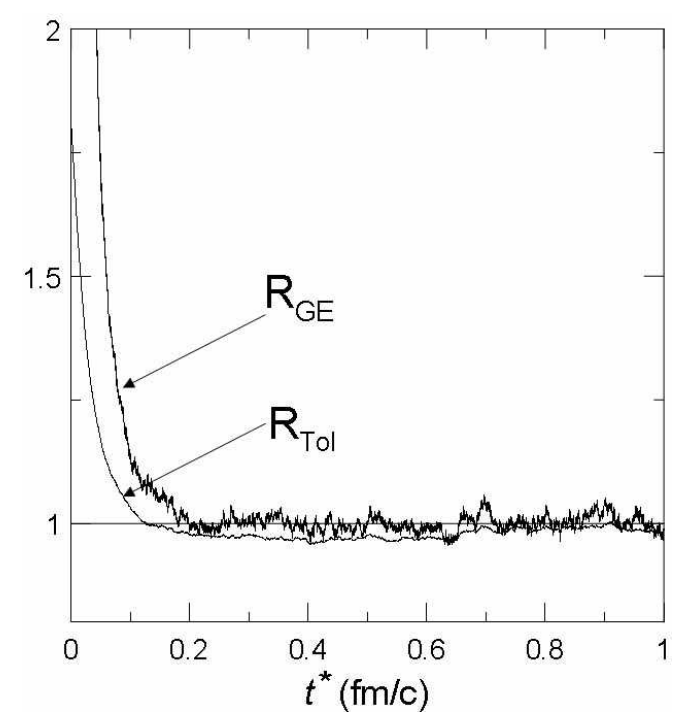

FIG. 2: The two ratios as a function of time $t^{*}$ with the initial condition of $300 \mathrm{MeV}$.

RIC physics. In Figs. 1 and 2, we plot the ratio,

$$
R_{\mathrm{GE}}\left(t^{*}\right)=\frac{1}{T} \frac{\left\langle D\left(p^{0 *}\right) \frac{\left(\mathbf{p}^{*}\right)^{2}}{\left(p^{0 *}\right)^{2}}\right\rangle_{t^{*}}}{\left\langle\nabla_{p} \cdot \frac{D\left(p^{0 *}\right) \mathbf{p}^{*}}{p^{0 *}}\right\rangle_{t^{*}}}
$$

by solving Eq. (37b) for one dimensional case with $\phi=0$. In this example, we compare the two different situations; one is that the pion is initially has a lower energy than the temperature of the heat bath, $p\left(t^{*}=0\right)=50 \mathrm{MeV}$ (Fig. 1), and the other the pion has initially a higher energy, $p\left(t^{*}=0\right)=300 \mathrm{MeV}$ (Fig. 2). When the null heat transfer $\left\langle d^{\prime} Q / d t^{*}\right\rangle_{\mathrm{RF}}=0$ is attained, we should have $R_{\mathrm{GE}}\left(t^{*}\right)=1$. For the sake of comparison, we show also the ratio

$$
R_{\mathrm{Tol}}\left(t^{*}\right)=\frac{1}{T}\left\langle\frac{p^{2}}{p^{0}}\right\rangle_{t^{*}},
$$

which corresponds to the Tolman relation for one dimensional case. In thermal equilibrium, this value should stay at $R_{\mathrm{Tol}}\left(t^{*}\right)=1$. In these simulations, we consider the case of $q=-1$, that is, $D\left(p^{0 *}\right)=K / p^{0 *}$ with $K=0.1(\mathrm{MeV})^{4}$. As seen from these figures, the null heat transfer condition is almost satisfied before $t^{*} \simeq 0.5 \mathrm{fm} / c$, whereas the Tolman relation only converges for $t^{*}>1 \mathrm{fm} / \mathrm{c}$. We checked some different parameter sets and found that such a behavior always appears. 


\section{SUMMARY AND CONCLUDING REMARKS}

In this paper, we extended the stochastic energetics for relativistic Brownian motion. As a model for the relativistic particle embedded in a heat bath, we first established a relativistic SDE which produces the invariant Jüttner equilibrium distribution under an arbitrary Lorentz transformation. Using this model, we discussed the thermodynamic laws and the equipartition theorem, by applying the stochastic energetics. We showed how the first and second laws of thermodynamics are derived from relativistic Brownian motion in this context. We obtained the explicit form of the heat transfer rate between the relativistic Brownian particle and the heat bath. Of course, our results recovers the corresponding results of Ref.[1] in the non-relativistic limit. As a result, we showed that the concept of the stochastic energetics is applicable to relativistic Brownian motion.

We further showed that the condition of the null heat transfer leads to the generalized equipartition theorem. Except for the particular choice of the parameters of the SDE, the generalized equipartition theorem does not coincide with Tolman's relativistic equipartition theorem if not in thermal equilibrium. The null heat transfer and thermal equilibrium are not equivalent, but the former includes the latter. As a matter of fact, we found that the generalized equipartion relation is attained before the system reaches the true thermal equilibrium in a few examples. It will be interesting to investigate more in detail the meaning of null heat transfer condition with respect to possible transient thermodynamic properties.

The stochastic energetics is considered as a promising approach for the study of thermodynamics of mesoscopic systems [1, 28]. Thus, the present study will be a starting point of the application of the stochastic energetics for the physics of relativistic heavy ion collisions. There, the finite size of the system, as well as the short reaction time are not negligible so that the deviation from the local thermal equilibrium must be clarified. In particular, the approach via the stochastic energetics allows introducing the energy conservation between microscopic and macroscopic degrees of freedom. This may serve for a consistent description of the formation of initial condition or the freezeout processes of final states in the hydrodynamic modeling [29].

The authors acknowledge stimulating discussions with G. S. Denicol. This work was (financially) supported by CNPq, FAPERJ, CAPES, PRONEX and the Helmholtz Interna- 
tional Center for FAIR within the framework of the LOEWE program (Landesoffensive zur Entwicklung Wissenschaftlich- Ökonomischer Exzellenz) launched by the State of Hesse.

\section{Appendix A: Ito formula}

Let us consider an arbitrary function $f(\mathbf{x})$ and the evolution of $\mathbf{x}$ is given by the SDE,

$$
d \mathbf{x}=\mathbf{A} d t+\mathbf{B} \circledast d \mathbf{w} .
$$

Then, the variation of $f(\mathbf{x})$ is

$$
d f(\mathbf{x})=\left\{\sum_{i} \mathbf{A}^{i} \partial_{i} f(\mathbf{x})+\frac{1}{2} \sum_{i j}\left[\mathbf{B B}^{T}\right]^{i j} \partial_{i} \partial_{j} f(\mathbf{x})\right\} d t+\sum_{i j} \mathbf{B}^{i j} \partial_{i} f(\mathbf{x}) \circledast d \mathbf{w}^{j}
$$

This is called the Ito formula [17]. By using the Ito formula, we obtain,

$$
\begin{aligned}
& {[\mathbf{B} \circ d \mathbf{w}]^{i}=[\mathbf{B} \circledast d \mathbf{w}]^{i}+\frac{1}{2} \sum_{j k} \mathbf{B}^{j k} \partial_{j} \mathbf{B}^{i k} d t,} \\
& {[\mathbf{B} \star d \mathbf{w}]^{i}=[\mathbf{B} \circledast d \mathbf{w}]^{i}+\sum_{j k} \mathbf{B}^{j k} \partial_{j} \mathbf{B}^{i k} d t .}
\end{aligned}
$$

Thus we can conclude as follows. When we have the Stratonovich-Fisk SDE,

$$
d \mathbf{x}=\mathbf{A} d t+\mathbf{B} \circ d \mathbf{w}
$$

this is equivalent to the Ito SDE,

$$
d \mathbf{x}^{i}=\left\{\mathbf{A}^{i}+\frac{1}{2} \sum_{j k} \mathbf{B}^{j k} \partial_{j} \mathbf{B}^{i k}\right\} d t+\mathbf{B} \circledast d \mathbf{w} .
$$

When we have the Hänggi-Klimontovich SDE,

$$
d \mathbf{x}=\mathbf{A} d t+\mathbf{B} \star d \mathbf{w}
$$

this is equivalent to the Ito SDE

$$
d \mathbf{x}^{i}=\left\{\mathbf{A}^{i}+\sum_{j k} \mathbf{B}^{j k} \partial_{j} \mathbf{B}^{i k}\right\} d t+\mathbf{B} \circledast d \mathbf{w}
$$

[1] K. Sekimoto, Stochastic Energetics (Springer, Berlin Heidelberg, 2010). 
[2] See, for example, R. Andrade, F. Grassi, Y. Hama, T. Kodama and O. . J. Socolowski, Phys. Rev. Lett. 97, 202302 (2006); J. Takahashi et al., Phys. Rev. Lett. 103, 242301 (2009); R. Andrade, F. Grassi, Y. Hama and W.-L. Qian, J. Phys. G37, 094043 (2010).

[3] R. M. Dudley, Arkiv Feur Mathematik 6, 241 (1965).

[4] R. Hakim, J. Math. Phys. 6, 1482 (1965).

[5] U. Ben-Ya'acov, Phys. Rev. D23, 1441 (1981).

[6] O. Oron and L. P. Horwitz, math-ph/0312003.

[7] T. H. Boyer, Phys. Rev. D19, 1112 (1979).

[8] T. H. Boyer, Phys. Rev. D19, 3635 (1979).

[9] A. Posilicano, Lett. Math. Phys. 42, 85 (1997).

[10] F. Debbasch, K. Mallick and J. P. Rivet, J. Stat. Phys. 88, 945 (1997).

[11] J. Franchi and Y. Le Jan, Comm. Pure Appl. Math. 60, 187 (2006).

[12] J. Dunkel and P. Hänggi, Phys. Rev. E71, 016124 (2005).

[13] J. Dunkel and P. Hänggi, Phys. Rev. E72, 036106 (2005).

[14] R. Zygadlo, Phys. Lett. A345, 323 (2005).

[15] J. Dunkel and P. Hänggi, Phys. Rep. 471, 1, (2009).

[16] J. Dunkel and P. Hänggi, Phys. Rev. E74, 051106 (2006).

[17] C. W. Gardiner, Handbook of Stochastic Method, (Springer, 2004).

[18] P. Hänggi, Hel. Phys. Acta 51, 183 (1078); ibid. 53, 491 (1980).

[19] P. Hänggi and H. Thomas, Phys. Rep. 88, 207 (1982).

[20] Y. Klimontovich, Phys. Usp. 37, 737 (1994).

[21] N. G. van Kampen, Physica 43, 244 (1969).

[22] F. Debbasch, J. P. Rivet and W. A. Leeuwen, Physica A301, 181 (2001).

[23] P.T. Landsberg and G.E.A. Matsas. Phys. Lett. A 223 (1996), p. 401.

[24] H. Spohn and J. L. Lebowitz, Adv. Chem. Phys. 38, 109 (1978).

[25] J. L. Synge, The Relativistic Gas (North-Hplland, Amsterdam, 1957).

[26] R. C. Tolman, Phys. Rev. 11, 261 (1918).

[27] A. Komer, Gen. Rel. Gra. 28, 379 (1996).

[28] See, for example, H. Kamegawa, T. Hondou and F. Takagi, Phys. Rev. Lett. 80, 5251 (1998); T. Houndou and K. Sekimoto, Phys. Rev. E62, 6021 (2000); U. Seifert, Phys. Rev. Lett. 95, $040602(2005)$. 
[29] H. Song, S. A. Bass, U. W. Heinz and T. Hirano, Chun Shen, arXiv:1101.4638 [nucl-th] and references therein.

[30] We use the notation $d^{\prime}$ for quantities which are, in general, not perfect differentials.

[31] Instead of introducing a scalar potential as in Eq.(37b), we may as well introduce it through the mass shift as $m \longrightarrow m-g \phi\left(\mathbf{x}^{*}, t^{*}\right)$, where $g$ is a coupling constant. This type of interaction is known, for example, in the relativistic mean-field theory in nuclear physics. Even if we use such a kind of potentials, it is still possible to derive Eq. (40). 